\title{
A Study on the Efficacy and Safety of a New Endoscopic Nasal Mask for Intravenous Anaesthesia With Respiratory Retention: a Randomized Controlled Trial
}

Bin Yu ( $\nabla$ yubin@tongji.edu.cn )

Department of Anesthesiology, Shanghai Tongji Hospital, Tongji University School of Medicine, Shanghai 200065, China

\section{Mengying Wang}

Department of Anesthesiology, Shanghai Tongji Hospital, Tongji University School of Medicine, Shanghai 200065, China

\section{Xinyu Cheng}

Department of Anesthesiology, Shanghai Tongji Hospital, Tongji University School of Medicine, Shanghai 200065, China

\section{Haiyue Ma}

Department of Anesthesiology, Shanghai Tongji Hospital, Tongji University School of Medicine, Shanghai 200065, China

\section{Shuqi Xie}

Department of Anesthesiology, Shanghai Tongji Hospital, Tongji University School of Medicine, Shanghai 200065, China

\section{Research Article}

Keywords: Intravenous anaesthesia, Respiratory retention, Endoscopic mask, Randomized controlled trial

Posted Date: March 3rd, 2021

DOl: https://doi.org/10.21203/rs.3.rs-254489/v1

License: (c) (1) This work is licensed under a Creative Commons Attribution 4.0 International License. Read Full License 


\section{Abstract}

Background: General anaesthesia is the most common choice for day surgery, and a laryngeal mask airway is the most commonly used airway management method in general anaesthesia. Many of the complications of laryngeal mask airways are not conducive to a rapid recovery and discharge. The main purpose of this study was to verify the effectiveness and safety of a new endoscopic nasal mask for anaesthesia for a short surgery.

Methods: This is a randomized controlled study of 96 patients who were scheduled to undergo hysteroscopy, breast mass resection or great saphenous vein lesion resection. They were randomly divided into two groups. After entering the operating room, 48 patients in the control group were treated with LMA-assisted ventilation, and 48 patients in the experimental group were subjected to assisted ventilation with the new endoscopic nasal mask. Both groups were given dexmedetomidine combined with sufentanil, propofol, dexamethasone, parecoxib sodium, dezocine and ramosetron to complete the intravenous general anaesthesia.

The main observation indexes included the occurrence of hypoxemia (SpO2<90\%) after anaesthesia induction; the occurrence of hypercapnia (PetCO2>50 mmHg) due to respiratory depression and changes in the $\mathrm{PaO} 2$ and $\mathrm{PaCO} 2$ values in the arterial blood gas analysis $(\mathrm{ABG})$ results.

Results: The occurrence of hypoxemia (SPO2 $<90 \%)$ in the experimental group and the control group was not significantly different $(P>0.05)$. The occurrence of hypercapnia (PetCO2>50 mmHg) in the experimental group was significantly reduced compared to that in the control group $(P<0.05)$, and the difference was statistically significant. According to the results of the preoperative and postoperative arterial blood gas analysis, there was no statistically significant difference in $\mathrm{PaO} 2$ and $\mathrm{PaCO} 2$ between the two groups $(P>0.05)$.

Conclusion: Both methods are safe and effective for anaesthesia during a short operation. The new endoscopic nasal mask can quickly and effectively provide effective ventilation . It is convenient for anaesthesia airway management for clinical short day surgery.

Trial registration: Trial Registration: www.chictr.org.cn; registration no.: ChiCTR2000032548; date of registration: 3rd May 2020

\section{Background}

Due to the progress in enhanced recovery after surgery (ERAS), day surgery is gradually becoming more common, and anaesthesia management has become a key component of ERAS. ERAS advocates the use of fast-track anaesthesia technology, which reduces the recovery time from anaesthesia and PACU residence time and reduces the related costs $\varangle 1 \bigotimes$. General anaesthesia is the most widely used anaesthesia mode in day surgery, and perioperative airway management is an important part of 
enhanced recovery after surgery. Generally, tracheal intubation, laryngeal mask airway or an oropharyngeal airway can be selected to maintain an unobstructed airway.

The objective of airway management during anaesthesia for day surgery is to maintain effective ventilation and oxygenation. The new endoscopic nasal mask used in this study is an innovative product that has been authorized with the invention patent (patent number: ZL201210286504.5) as shown in Fig. 1 and Fig. 2. The use of the new endoscopic nasal mask can allow for the patient's spontaneous breathing while keeping the airway unobstructed. It can also be connected to a simple breathing apparatus or an anaesthesia machine through the side hole to provide a continuous oxygen supply or a pressurized oxygen supply to assist breathing $₫ 2 \rrbracket$. The purpose of this study was to explore a safe, effective, convenient and easy to popularize method of airway management under anaesthesia in a short surgery by using a new endoscopic nasal mask.

\section{Materials And Methods Study design}

This randomized, controlled trial was approved by the ethics committee of our hospital.

The product has previously been used in fiberoptic bronchoscope-guided endotracheal intubation and

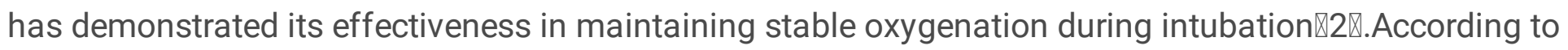
Ehrenfeld $₫ 3$ \et al., the occurrence of hypoxemia after anaesthesia induction is $7.4 \%$. In this study, the occurrence of hypoxemia after anaesthesia induction with positive control devices was conservatively considered to be $17.4 \%$. The default non-inferiority threshold was $10 \%$ and it was assumed that the difference in the true effective rate between the control group and the experimental group was 0 . PASS15 was used to calculate the minimum sample size of 42 patients in each group, indicating a total of 96 patients should be included in this study plan with 48 patients in each group at a $15 \%$ drop-off rate. The patients and their family members signed the informed consent before the operation. During the preoperative visit, preoperative education was carried out to introduce the operation process, the anaesthesia methods and the precautions, to reduce the psychological worries and fears of the patients. At the same time, the consultation questions of the patients were answered in detail.And all procedures have been approved by the hospital as safe and effective.All the methods used in this study were in accordance with the regulations of the Institutional Review Ethics Committee of the author's institution, and all the methods (including case selection, specimen collection and data analysis, etc.) comply with the procedures and regulations of Chinese clinical trials.

\section{Participants}

The subjects were recruited from patients who underwent hysteroscopy, breast mass resection or great saphenous vein lesion resection in our hospital from May 2020 to October 2020. The inclusion criteria included ASA I to II grade, ages 18 to 70 years. Patients with an active upper respiratory tract infection or pneumonia, potential respiratory tract obstruction, structural deformity of the oropharynx, airway injury, 
acute pharyngitis, chronic respiratory disease, airway hyperresponsiveness, coagulation dysfunction, and pregnant or lactating women were excluded.

\section{Methods}

The patients were divided into two groups according to the random number table method.

Then, 48 patients in the control group were treated with LMA assisted ventilation and 48 patients in the experimental group were treated with the new endoscopic nasal mask assisted ventilation. Both groups were given dexmedetomidine combined with sufentanil, propofol, dexamethasone, parecoxib sodium, dezocine and ramosetron to complete intravenous general anaesthesia(Figure 3).

The patient fasted at least for 8 hours before the operation. After entering the operating room, noninvasive blood pressure, pulse oximetry and electrocardiogram were routinely monitored, and oxygen was inhaled by a nasal catheter at $2 \mathrm{~L} / \mathrm{min}$. One side of the peripheral vein of the upper limb was opened, a colloid was injected into the vein for dilation, and arterial blood was extracted for arterial blood gas analysis (ABG).

Anaesthesia induction method: dexmedetomidine was injected intravenously for sedation. The loading dose of dexmedetomidine was $1 \mu \mathrm{g} / \mathrm{kg}$ for 10 minutes, and then adjusted to $0.3 \mu \mathrm{g} / \mathrm{kg} / \mathrm{h}$.

Dexamethasone $5 \mathrm{mg}$, parecoxib sodium $40 \mathrm{mg}$ and dezocine $5 \mathrm{mg}$ were injected intravenously before the operation. Propofol was maintained at $60 \mathrm{mg} / \mathrm{kg} / \mathrm{h}$ after anaesthesia induction. Both groups were given ramosetron $0.3 \mathrm{mg}$ intravenously at the end of the operation and a second arterial blood gas analysis (ABGs) was performed 30 minutes after the end of anaesthesia.

Experimental group operation method: The patient was placed in a prone position and intravenously injected with $0.2-0.4 \mu \mathrm{g} / \mathrm{kg}$ sufentanil and $1.5-2.0 \mathrm{mg} / \mathrm{kg}$ propofol, and after sufficient sedation and analgesia, we fastened the mask to allow the patient to fully breathe oxygen and then slightly tilted their head back while maintaining the three axes of the respiratory tract (mouth, pharynx, and larynx) in the same straight line as much as possible to keep the upper airway unobstructed. The pharyngeal curvature of the oropharyngeal airway was sent to the upper pharynx along the tongue surface, and the tongue base was separated from the oropharyngeal posterior wall. Then, the oropharyngeal airway was inserted into the corresponding slot of the mask and the mask was connected to the respiratory circuit to ensure the oxygen supply. The oxygen-flow rate was set at 3-5 L/min, allowing for the patient's spontaneous breathing during the whole process. Propofol could be slowly injected intravenously when the patient had body movements, coughing, etc. In cases of respiratory depression, manual assisted breathing and pressure-supported ventilation modes could be used for respiratory support until spontaneous respiration is restored. When SpO2 $<90 \%$ occurs, the first step at this point is to hold the jaw by the hand, open the airway, and continuously provide oxygen with the mask. If SpO2 $>95 \%$, mask ventilation is proven to be effective. If the ventilation is still poor, mechanical ventilation through a hand mask is provided in the second step. If SpO2 $>95 \%$, the mask ventilation is proven to be effective. If the SpO2 is still below $90 \%$, we need place a laryngeal mask, and the ventilation of the new endoscopic nasal mask is considered to 
have failed at this time. If the ventilation continues to be poor, in order to ensure the patient's safety, the patient should be intubated under general anaesthesia at this time, and the case is considered excluded.

Operation methods in the control group: The patient was placed prone and intravenously injected with $0.2-0.4 \mu \mathrm{g} / \mathrm{kg}$ sufentanil and $1.5-2.0 \mathrm{mg} / \mathrm{kg}$ propofol and after sufficient sedation and analgesia, we fasten the mask to make the patient fully breathe oxygen, and then we slightly tilt the head back and the operator pulls the lower jaw with the left hand to widen the oral space and holds the laryngeal mask airway (LMA) with the right hand. With the mouth of the LMA facing the lower jaw, the laryngeal mask is inserted along the midline of the tongue to the posterior pharyngeal wall until it could not be pushed further forward. After the LMA laryngeal mask airway is confirmed to be effective, we connect the LMA with the respiratory circuit, and the oxygen-flow rate is set at $3 \sim 5 \mathrm{~L} / \mathrm{min}$. The position of the laryngeal mask airway is fixed with adhesive tape, and the patient is allowed to breathe autonomously. Propofol can be slowly injected intravenously when the patient has body movements, coughing, and other forms of movement.In cases of poor ventilation, manual assisted breathing and pressure-supported ventilation modes can be used for respiratory support until spontaneous respiration is restored. When $\mathrm{SpO} 2<90 \%$ occurs, the first step is to hand-squeeze the anaesthesia machine breathing balloon to provide assisted breathing at this point. If SpO2 >95\%, LMA ventilation is proven to be effective. If SpO2 is still below 90\%, mechanical ventilation can be used and if $\mathrm{SpO} 2>95 \%$, LMA ventilation is proven to be effective. If ventilation continues to be poor, in order to ensure the patient's safety, the patient should be intubated under general anaesthesia at this time, and the case is considered excluded.

Both groups were treated by the same anaesthesiologist. Atropine was given if the heart rate (HR) dropped to less than 50 times/min. If the blood pressure was higher or lower than $30 \%$ of the basal blood pressure, nitroglycerine, deoxyepinephrine, ephedrine, etc., should be given to maintain a stable blood pressure.

\section{Observation index}

The main observation indexes included the occurrence of hypoxemia $(\mathrm{SpO} 2<90 \%)$ after anaesthesia induction, the occurrence of hypercapnia (PetCO2 $>50 \mathrm{mmHg}$ ) due to respiratory depression and the changes of $\mathrm{PaO} 2$ and $\mathrm{PaCO} 2$ in the two arterial blood gas analysis (ABG) results.

The secondary observation indexes included the airway placement time, the success rate of primary catheterization and the occurrence of adverse reactions in the patients. The adverse reactions in the patients in the two groups were compared, and the complications including sore throat, hoarseness, laryngeal spasm, reflux and aspiration, pneumonia, atelectasis of the lung, and re-expansion pulmonary oedema.

\section{Statistical analyses}

Statistical analyses were performed using SPSS version 26.0 (SPSS Inc., Chicago, IL). The continuous data were expressed as the mean \pm standard deviation ( $x \pm s)$, and t-tests were used for the comparisons 
between the groups. The counting data were expressed as rate (\%) and the rank-sum test was used. $\mathrm{P}<$ 0.05 was considered statistically significant.

\section{Results}

\section{Clinical characteristics of the patients}

There was no statistically significant difference between the two groups in terms of gender, age, BMI, ASA grade or any other of the general conditions $(P>0.05)$. The baseline characteristics of the patients have been reported in Table 1.

Table 1

Comparison of the clinical characteristics

\begin{tabular}{|lllll|}
\hline & Male/Female & Age $(\mathrm{y})$ & $\mathrm{BMI}\left(\mathrm{kg} / \mathrm{m}^{2}\right)$ & ASA ((I/II) \\
\hline Experimental group & $2 / 46$ & $40.48 \pm 12.07$ & $22.00 \pm 3.36$ & $37 / 11$ \\
Control group & $0 / 48$ & $37.17 \pm 10.30$ & $22.83 \pm 3.37$ & $41 / 7$ \\
\hline
\end{tabular}

\section{The occurrence of hypoxemia and hypercapnia}

There was no significant difference in the occurrence of hypoxemia between the experimental group and the control group. By the rank-sum test comparison, $Z=0.798, P=0.425>0.05$, and the difference was not statistically significant. The occurrence of hypercapnia in the experimental group was significantly lower than that in the control group. By the rank-sum test, $\mathrm{Z}=2.728, \mathrm{P}=0.006<0.05$, and the difference was statistically significant as shown in Table 2.

Table 2

The occurrence of hypoxemia and hypercapnia

\begin{tabular}{|lll|}
\hline & Hypoxemia & Hypercapnia \\
\hline & 0 time 1 time & 0 time 1 time 2 times 3 times 4 times \\
\hline Experimental group & $38(79.2 \%) 10(20.8 \%)$ & $13(27.1 \%) 25(52.0 \%) 7(14.6 \%) 2(4.2 \%) 1(2.1 \%)$ \\
Control group & $41(85.4 \%) 7(14.6 \%)$ & $8(16.7 \%) 15(31.2 \%) 18(37.5 \%) 6(12.5 \%) 1(2.1 \%)$ \\
Statistics & $\mathrm{Z}=0.798, \mathrm{P}=0.425$ & $\mathrm{Z}=2.728, \mathrm{P}=0.006$ \\
\hline
\end{tabular}

The success rate of primary catheterization and the airway placement time

The success rate of primary catheterization in the experimental group was significantly higher than that in the control group. After applying the rank-sum test, $Z=3.854, P<0.001$, the difference was statistically significant.A comparison of the airway placement time between the two groups showed a statistically significant difference $(P<0.001)$ as shown in Table 3. 
Table 3

The success rate of primary catheterization and the airway placement time

\begin{tabular}{|lll|}
\hline & Intubation attempts & Placement time \\
\hline & 1 time 2 times 3 times & Average $((\mathrm{s})$ \\
\hline Experimental group & $48(100 \%) 0(0.0 \%) 0(0.0 \%)$ & $5.33 \pm 2.25$ \\
Control group & $35(72.9 \%) 12(25 \%) 1(2.1 \%)$ & $15.94 \pm 16.78$ \\
Statistics & $\mathrm{Z}=3.854 \mathrm{p}<0.001$ & $\mathrm{t}=4.34 \mathrm{p}=0.00$ \\
\hline
\end{tabular}

The changes in PaCO2 in the arterial blood gas analysis (ABG)

As shown in Fig. 4, a comparison of the change of $\mathrm{PaCO} 2$ in the two arterial blood gas analyses between the two groups showed no statistical significance $(P>0.05)$.

\section{The changes in $\mathrm{PaO} 2$ in the arterial blood gas analysis (ABG)}

As reported in Fig. 5, the changes in $\mathrm{PaO} 2$ in the two arterial blood gas analyses between the two groups were compared, and the difference was not statistically significant $(P>0.05)$.

\section{Discussion}

Enhanced Recovery After Surgery (ERAS) refers to the adoption of a series of optimized perioperative management measures to accelerate the recovery of patients, reduce their mortality and postoperative complications, reduce hospitalization costs and shorten postoperative hospital stays, thus improving the patient's prognosis $₫ 4$.

Day surgery, also known as ambulatory surgery, refers to a clinical treatment mode that can be completed within 24 hours after admission, including diagnosis and treatment (surgery or operation) and discharge from the hospital by optimizing the diagnosis and treatment process for surgical diseases that used to require hospitalization for several days. The concept of ambulatory surgery was first proposed by Dr. Nicoll in England. With the establishment of The International Association of Ambulatory Surgery (IAAS), ambulatory surgery has developed into a more mature mode of operation management $\mathbb{5}$ \.

Day surgery allows patients to return to their normal life earlier by simplifying admission procedures and shortening their length of stay. Through the implementation of ERAS, the goal of shortening hospitalization time and reducing medical costs is exactly in line with the goals of day surgery.

General anaesthesia and intraspinal anaesthesia are commonly used in day surgery. However, both spinal anaesthesia and epidural anaesthesia may cause urinary retention, and patients need a complete return of their sensory and motor functions of the lower extremities before they can go home, and complications such as intraspinal infection and bleeding may occur within days after surgery. Therefore, 
these two methods of anaesthesia are generally not preferred during day surgery. General anaesthesia is the most widely used anaesthesia method in day surgery.

Tracheal intubation, a laryngeal mask airway, or an oropharyngeal airway can be generally selected for airway management to maintain an unobstructed airway. The study by Doksrod $\llbracket 6$ \et al. showed that compared with endotracheal intubation, a laryngeal mask airway (LMA) could appropriately reduce the dosage of anaesthetics and it can be successfully placed without the use of skeletal muscle relaxants, which can accelerate the recovery of muscle strength and the recovery of the patients, reduce the violent fluctuation of haemodynamics during the induction and the recovery period, and avoid the excessive use of skeletal muscle relaxants and antagonists. In addition, laryngeal mask airway can also complete the anaesthesia while retaining the patient's spontaneous breathing $\nabla 7 \varnothing$.

Laryngeal mask airway (LMA) is a kind of ventilation device between endotracheal intubation and mask oxygen inhalation. It is convenient, safe and effective for the establishment of an artificial airway during general anaesthesia, and therefore, laryngeal mask airway anaesthesia is one of the commonly used anaesthesia methods during short surgery. However, when the laryngeal mask airway enters the larynx, the laryngeal mucosa and vocal cords may be damaged, and compression of the laryngeal mask airway may cause varying degrees of inflammatory damage and oedema to the larynx and vocal cords. Therefore, patients are prone to have adverse reactions such as a sore throat and hoarseness after the operation.Second, improper pressure of the laryngeal mask airway and a long indwelling time can increase the incidence of postoperative pharyngeal complications after the application of a laryngeal mask airway $\mathbb{8}$. Studies by Choo $₫ 9$ \et al. found that the incidence of laryngopharyngeal pain was approximately $35.2 \%$ after a classic laryngeal mask insertion. Enrique Gorb囚10》et al. have reported cases of bilateral vocal cord dysfunction after laryngeal mask airway general anaesthesia. In addition, laryngeal mask general anaesthesia needs to maintain a certain depth of anaesthesia. Under shallow anaesthesia, laryngeal spasms can easily occur, and it is also easy to cause intraoperative awareness, increase the patient's pain, and cause haemodynamic instability. However, deep anaesthesia may lead to drug accumulation and prolong the recovery time of patients, which is contrary to the concept of Enhanced Recovery After Surgery. It can be seen that there are still many complications when a laryngeal mask airway is used for airway management of general anaesthesia.

In this study, dexmedetomidine, sufentanil, propofol, dexamethasone, parecoxib sodium, dezocine and ramosetron were combined for complete intravenous general anaesthesia, and patients retained the ability to breathe on their own, which allowed for a safe, effective, convenient and easily promoted airway management method after anaesthesia for day surgery.

In this study, a new endoscopic mask was used and compared with an LMA laryngeal mask. The results showed that in terms of airway placement, the success rate of primary placement in the control group was $72.9 \%$ as shown in Table 3 and that in the experimental group was $100 \%$, which was significantly better than the control group $(P<0.001)$, and the time required for placing the mask in the experimental group ( $(5.33 \pm 2.25)$ was shorter than that of the control group $(15.94 \pm 16.78)$. This is because the new 
endoscopic nasal mask can be applied to the face of the patient after the matching oropharyngeal airway is inserted into the oropharyngeal cavity, combined with the nasal mask rotation card, and then the nasal mask can be fixed onto the face of the patient with four headbands. Meanwhile, an LMA laryngeal mask airway (LMA) needs to be placed in the supraglottic position and is susceptible to laryngopharyngeal anatomic variations, which increases the incidence of secondary catheterization. This indicates that compared with the LMA laryngeal mask airway, the new endoscopic nasal anaesthesia is more convenient and quicker to place during an operation.

In terms of airway safety, there was no significant difference in the occurrence of hypoxemia (Spo2< $90 \%)((P=0.425>0.05)$ between the two groups after anaesthesia induction, and the occurrence of hypercapnia (PetCO2 $>50 \mathrm{mmHg}$ ) in the experimental group was significantly lower than that in the control group $(P=0.006<0.05)$ as shown in Table 2 , and the difference was statistically significant. This is because the new endoscopic mask can effectively prevent any respiratory tract obstruction caused by the backdrop of the tongue base and keep the patient breathing autonomously while keeping the airway unobstructed.

According to the results of the preoperative and postoperative arterial blood gas analysis as shown in Fig. 3 and Fig. 4, there was no statistically significant difference in $\mathrm{PaO} 2$ and $\mathrm{PaCO} 2$ between the two groups ( $(P>0.05)$; this is due to the only small amount of narcotic drugs necessary we use the new endoscopic mask. It is not easy to cause drug accumulation, and the patients can wake up rapidly after the operation, which is conducive to the early and rapid recovery of patients. In addition, there was no statistically significant difference in the incidence of adverse reactions between the two groups $(P>0.05)$, showing that the new endoscopic mask can greatly reduce the incidence of oropharynx mucosa damage and throat pain, and avoid the haemodynamic changes caused by laryngeal mask stimulation. All of these findings have proven that the new endoscopic nasal mask is effective and safe for ventilation during intravenous anaesthesia.

\section{Conclusion}

Both the LMA laryngeal mask and the new endoscopic nasal mask can be used safely and effectively for intravenous anaesthesia that preserves the patient's spontaneous breathing. The new endoscopic nasal mask has the advantages of more convenient operation, reduced throat irritation, fewer throat complications, a stable ventilation effect and improved patient comfort, which is worthy of promotion and application in short day surgery.

\section{Declarations}

\section{Funding information}

This work was supported by Shanghai Municipal Commission of Science and Technology 『NO:18441905700区 


\section{Competing interests}

The authors report no conflicts of interest concerning the materials or methods used in this study or the findings specified in this paper.

\section{Availability of data and material}

All patients which were included in the present study were gave written informed consent for their data in this study. All data was obtained from the clinical and laboratory results records. This study was approved by the Institutional Review Ethics Committee of the authors' institution. All patients underwent surgical treatment in our hospital, and their data were compared before and after surgery. All data generated or analysed during this study are included in this published article.

\section{Trial registration: Trial Registration: www.chictr.org.cn; registration no.: ChiCTR2000032548; date of registration: 3rd May 2020}

\section{Code availability}

SPSS 250 statistical software package were used to edit the manuscript.

\section{Authors' contributions}

Study design: Yu Bin

Data collection: Cheng Xinyu ,Ma Haiyue ,Xie Shuqi

Statistical analysis: Wang Mengying

Manuscript preparation: Wang Mengying

Literature search: Wang Mengying

Funds collection: Yu Bin

\section{Authors'information}

Wang Mengying ${ }^{1} \mathrm{MD}$, Cheng Xinyu ${ }^{1} \mathrm{MD}, \mathrm{Ma}$ Haiyue ${ }^{1} \mathrm{MD}$, Xie Shuqi ${ }^{1} \mathrm{MD}$, Yu Bin * PhD

1 Department of Anesthesiology, Shanghai Tongji Hospital, Tongji University School of Medicine, Shanghai 200065, China

* Corresponding author:Yu Bin PhD,E-mail: yubin@tongji.edu.cn

Tel: +86-13918108880 Department of Anesthesiology, Shanghai Tongji Hospital, Tongji University School of Medicine, Shanghai 200065, China 


\section{References}

1. White PF, Kehlet H, Neal JM, Schricker T, Carr DB, Carli F; Fast-Track Surgery Study Group. The role of the anesthesiologist in fast-track surgery: from multimodal analgesia to perioperative medical care. Anesth Analg. 2007 Jun;104(6):1380-96, table of contents. doi:

10.1213/01.ane.0000263034.96885.e1. PMID: 17513630.

2. Zou T, Huang Z, Hu X, Cai G, He M, Wang S, Huang P, Yu B. Clinical application of a novel endoscopic mask: a randomized controlled, multi-center trial in patients undergoing awake fiberoptic bronchoscopic intubation. BMC Anesthesiol. 2017 Jun 15;17(1):79. doi: 10.1186/s12871-017-0370-y. PMID: 28619016; PMCID: PMC5472943.

3. Ehrenfeld JM, Funk LM, Van Schalkwyk J, Merry AF, Sandberg WS, Gawande A. The incidence of hypoxemia during surgery: evidence from two institutions. Can J Anaesth. 2010 Oct;57(10):888-97. doi: 10.1007/s12630-010-9366-5. Epub 2010 Jul 31. PMID: 20680710; PMCID: PMC2991088.

4. Varadhan $\mathrm{KK}$, Lobo DN, Ljungqvist $\mathrm{O}$. Enhanced recovery after surgery: the future of improving surgical care. Crit Care Clin. 2010 Jul;26(3):527-47, x. doi: 10.1016/j.ccc.2010.04.003. PMID: 20643305.

5. Cadariu F, Enache A, Avram M, Muresan C, Olariu S. Day surgery in Romania. Ann Ital Chir. 2017;88:567-571. PMID: 29339592.

6. Doksrod S, Lofgren B, Nordhammer A, Svendsen MV, Gisselsson L, Raeder J. Reinforced laryngeal mask airway compared with endotracheal tube for adenotonsillectomies. Eur J Anaesthesiol. 2010 Nov;27(11):941-6. doi: 10.1097/EJA.0b013e32833d69c6. PMID: 20739893.

7. Williams DL, Zeng JM, Alexander KD, Andrews DT. Randomised Comparison of the AMBU AuraOnce Laryngeal Mask and the LMA Unique Laryngeal Mask Airway in Spontaneously Breathing Adults. Anesthesiol Res Pract. 2012;2012:405812. doi: 10.1155/2012/405812. Epub 2012 Feb 29. PMID: $22505884 ;$ PMCID: PMC3299248.

8. Aydogmus MT, Eksioglu B, Oba S, Unsal O, Türk HS, Sinikoglu SN, Tug A. Comparison of laryngeal mask airway supreme and laryngeal mask airway proseal for laryngopharyngeal trauma and postoperative morbidity in children. Braz J Anesthesiol. 2013 Nov-Dec;63(6):445-9. doi: 10.1016/j.bjane.2012.08.004. Epub 2013 Dec 5. PMID: 24565340.

9. Choo CY, Koay CK, Yoong CS. A randomised controlled trial comparing two insertion techniques for the Laryngeal Mask Airway Flexible in patients undergoing dental surgery. Anaesthesia. 2012 Sep;67(9):986-90. doi: 10.1111/j.1365-2044.2012.07167.x. Epub 2012 Jun 11. PMID: 22686571.

10. Gorbea E, Mori M. Two Cases of Bilateral Vocal Fold Mobility Impairment After LMA Use In 7 Months. Ann Otol Rhinol Laryngol. 2018 Sep;127(9):653-656. doi: 10.1177/0003489418784061. Epub 2018 Jul 26. PMID: 30047792.

\section{Figures}




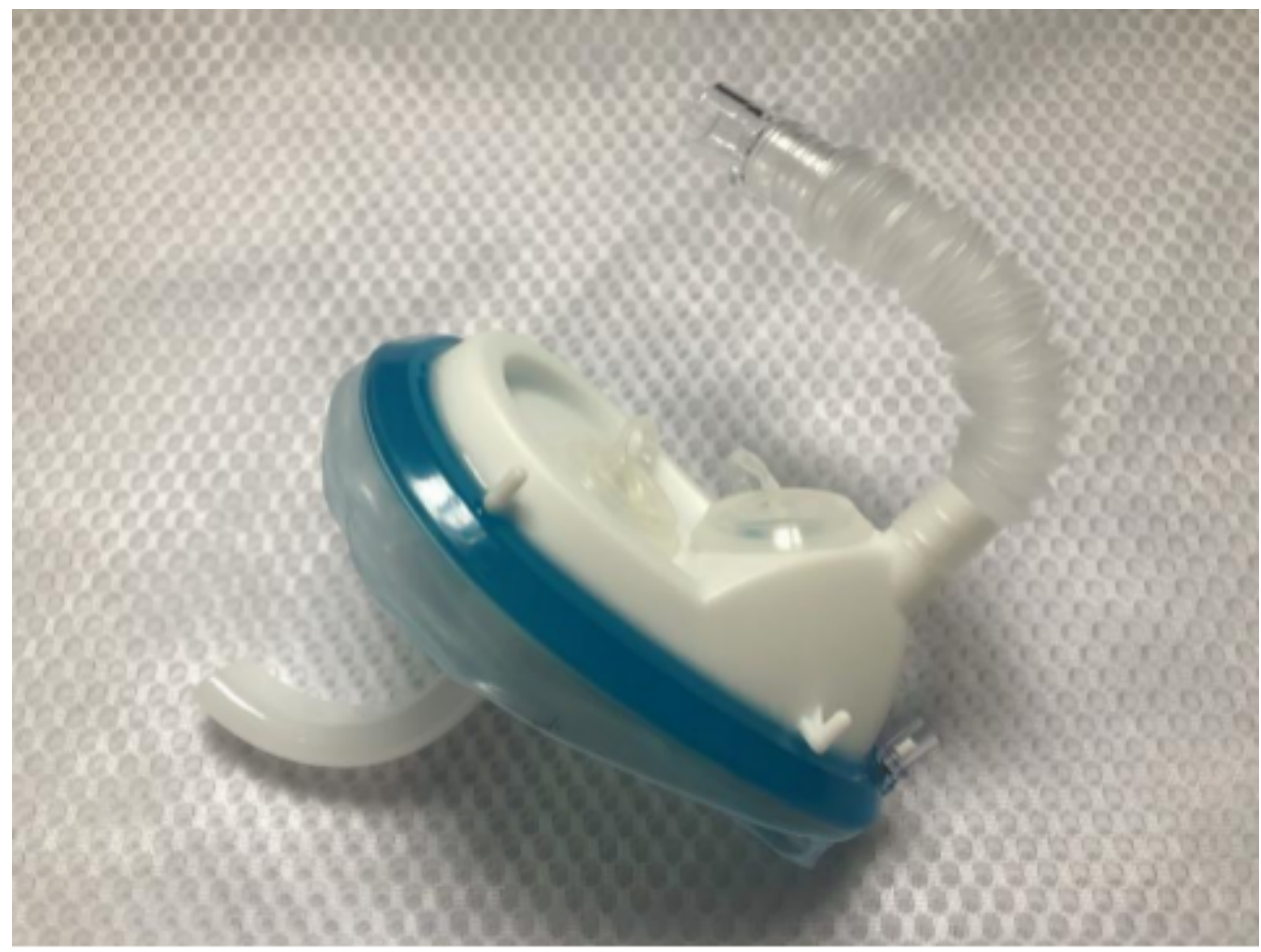

\section{Figure 1}

The new endoscopic nasal mask used in this study 


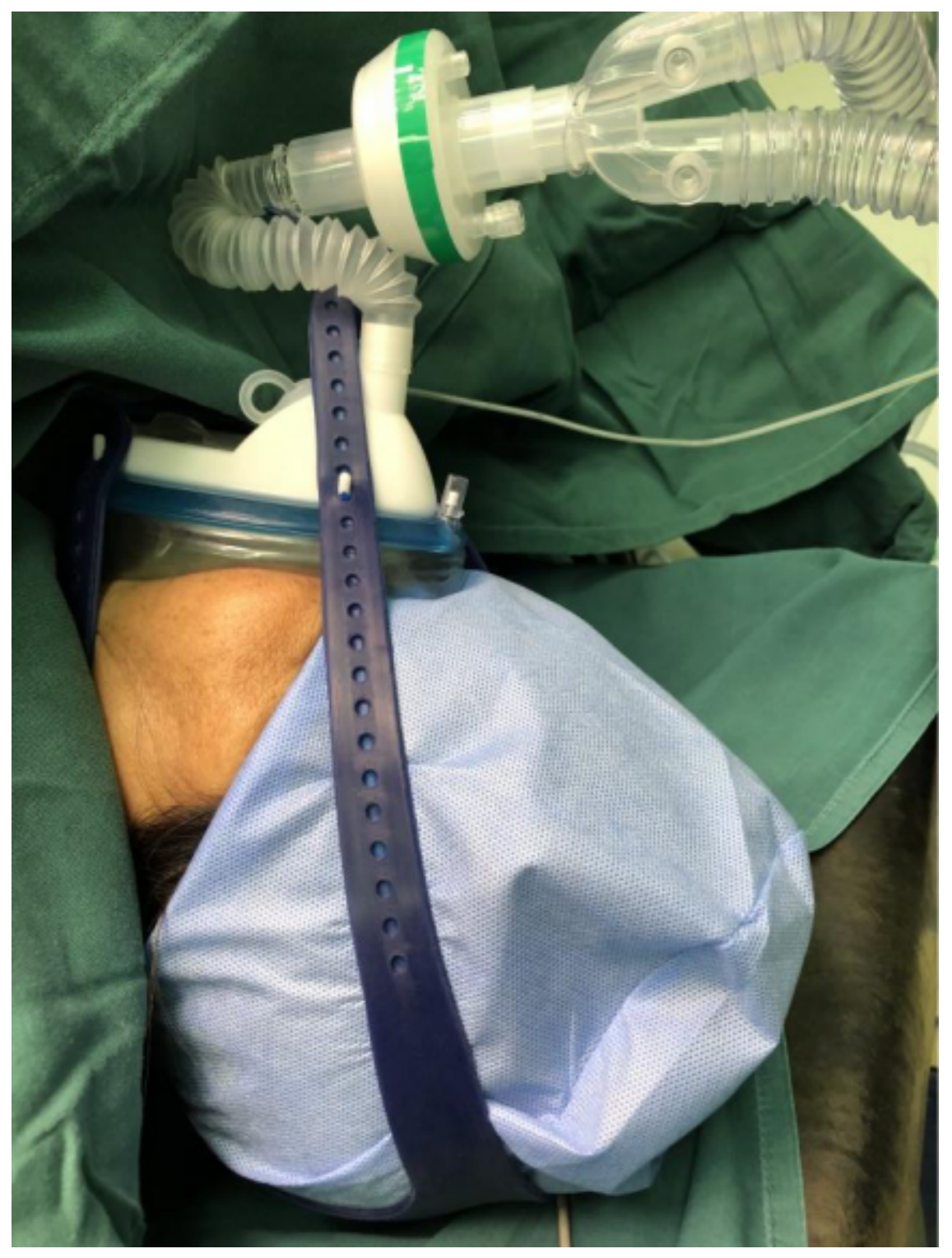

Figure 2

The new endoscopic nasal mask used in anaesthesia airway management of a breast mass resection 


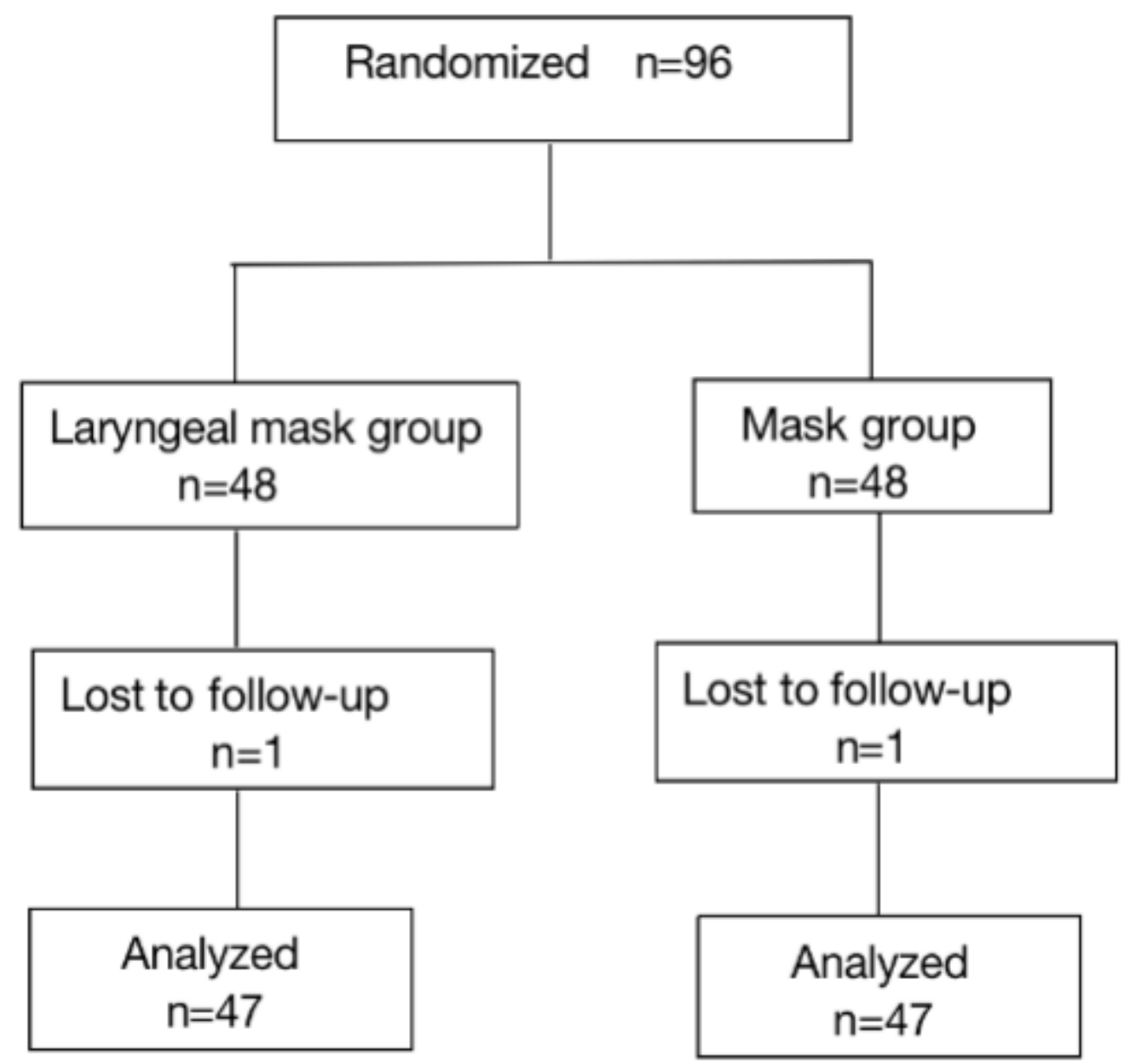

Figure 3

Flow chart of participant selection for the study

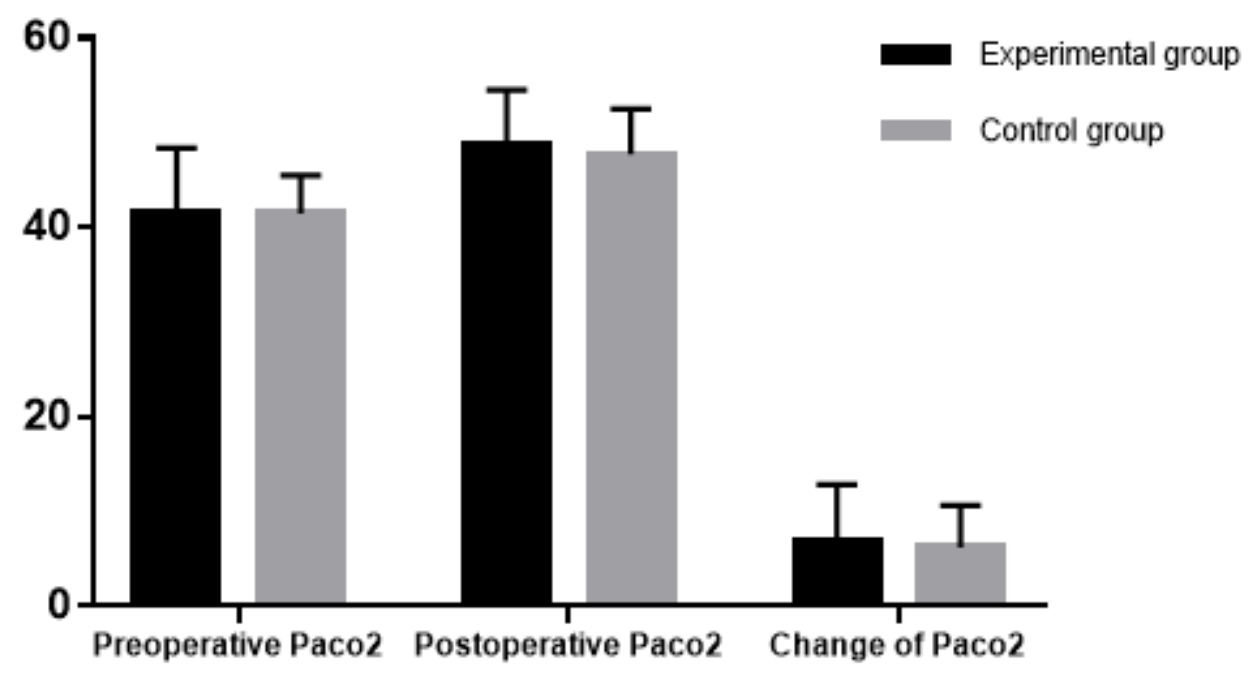

Figure 4 
Changes of $\mathrm{PaCO} 2$ in $\mathrm{ABG}$ (Comparison of $\mathrm{PaCO} 2$ before the operation between the two groups $\mathrm{p}=$ 0.882 , PaCO2 after the operation $p=0.437$, PaCO2 changes $p=0.518$ )

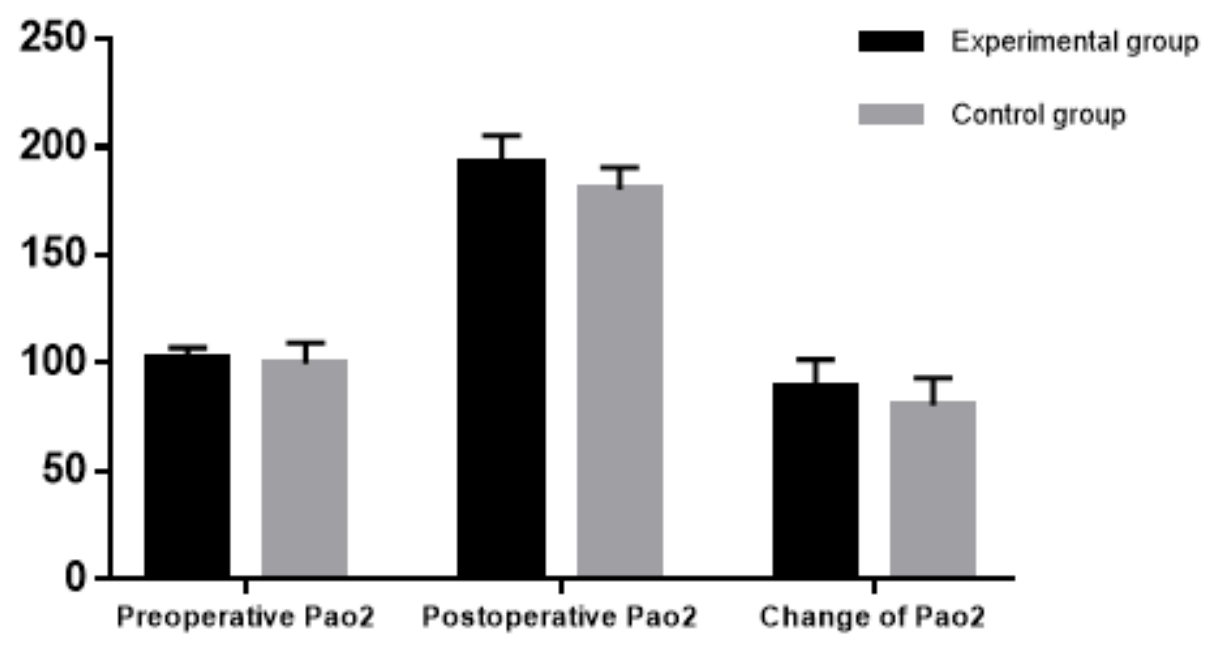

Figure 5

Changes of $\mathrm{PaO} 2$ in the $\mathrm{ABG}$ (Comparison of $\mathrm{PaO} 2$ before the operation between the two groups $\mathrm{p}=$ $0.832, \mathrm{PaO} 2$ after the operation $\mathrm{p}=0.441, \mathrm{PaO} 2$ changes $\mathrm{p}=0.519$ ) 